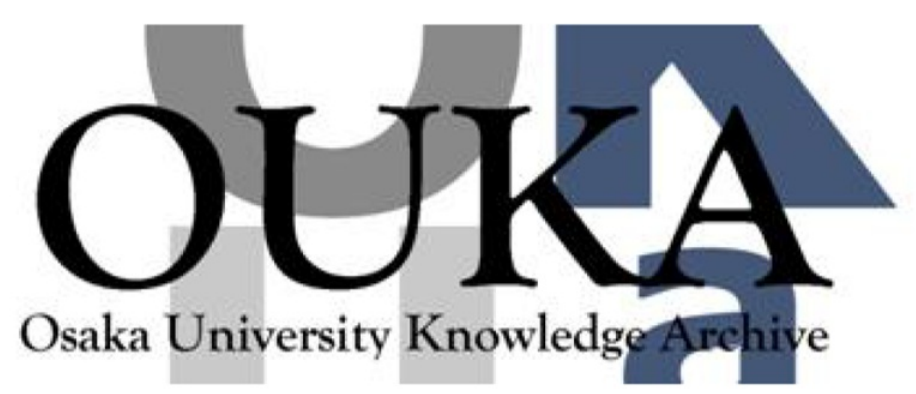

\begin{tabular}{|c|l|}
\hline Title & $\begin{array}{l}\text { Near-infrared ps transient photoinduced } \\
\text { absorption in conjugated systems }\end{array}$ \\
\hline Author(s) & $\begin{array}{l}\text { Frolov, Sergey V.; Gellermann, Werner; Vardeny, } \\
\text { Z. Valy et al. }\end{array}$ \\
\hline Citation & $\begin{array}{l}\text { Proceedings of SPIE - The International Society } \\
\text { for Optical Eng ineering. 3145 p. 453-p. 458 }\end{array}$ \\
\hline Issue Date & $1997-12-01$ \\
\hline oaire:version & VoR \\
\hline URL & https://hdl. handle. net/11094/76953 \\
\hline rights & \\
\hline Note & \\
\hline
\end{tabular}

Osaka University Knowledge Archive : OUKA

https://ir. Library. osaka-u. ac. jp/

Osaka University 


\title{
Near-Infrared ps Transient Photoinduced Absorption in Conjugated Systems.
}

\author{
S.V.Frolov ${ }^{\mathrm{a}}$, W. Gellermann ${ }^{\mathrm{a}}$, Z.V.Vardeny ${ }^{\mathrm{a}}$, M.Ozaki ${ }^{\mathrm{b}}$, K.Yoshino ${ }^{\mathrm{b}}$, T.Barton ${ }^{\mathrm{c}}$ and G.Lanzani ${ }^{\mathrm{d}}$ \\ aPhysics Department, University of Utah, Salt Lake City, UT 84112 \\ 'Electronic Engineering Department, Osaka University, Yamada-Oka 2-1 Suita, Japan \\ 'Ames Laboratory, Iowa State University, Ames, IA 50011-3160 \\ ${ }^{\mathrm{D} D i p a r t i m e n t o ~ d i ~ F i s i c a, ~ P o l i t e c n i c o ~ d i ~ M i l a n o, ~} 20133$ Milano, Italy
}

\begin{abstract}
We demonstrate the use of a color center laser in a pump-and-probe correlation technique which enables us to extend the probe spectral range into the infrared. As a result, we find strong photoinduced infrared absorption in several $\pi$-conjugated polymers and oligomers. We associate this absorption with optical transitions in the singlet exciton manifold.
\end{abstract}

Keywords: ultrafast spectroscopy, color center laser, conducting polymers, photoinduced absorption, singlet excitons, even and odd parity states.

\section{INTRODUCTION}

Excited state properties of $\pi$-conjugated polymers and oligomers are now the subject of intense experimental and theoretical studies. These conjugated systems promise to be the building blocks of new fully organic opto-electronic devices, such as light-emitting diodes, photocells, laser-diodes, thin-film transistors, etc. ${ }^{1}$ Therefore, understanding the photophysics of these materials is an important step in the development of new technologies based on organics. The well-known pumpand-probe ultrafast spectroscopy is an excellent tool for investigating the excited states properties. Its particular advantage is the ability to probe photoinduced absorption (PA) in the optical spectral range away from the fundamental absorption, which allows to distinguish between the photoresponse of the excited and ground states, respectively. The transient PA bands in $\pi$ conjugated systems extend deep into the infrared spectral range (IR). In particular, charge excitations, such as polarons and bipolarons, have strong optical transitions in the near and mid-IR ${ }^{2}$. However, a typical ps laser system spectral range covers only the visible part of the spectrum. It is very important to have a pulsed IR probe to conduct a time-resolved PA study of $\pi$ conjugated polymers and oligomers over a much broader spectral range.

We report a study of transient IR PA in several conducting polymers and an oligomer using a pulsed color center laser $(\mathrm{CCL})^{3}$ as a probe source for the time-resolved picosecond pump-and-probe correlation technique. Both groups of conducting polymers have been studied: luminescent conducting polymers (LCP), including poly(2,5-dioctyloxy-pphenylene vinylene) (DOO-PPV), poly(3-butyl thiophene) (P3BT) and poly(p-phenylene ethynylene) (PPE), and nonluminescent conducting polymers (NCP), including poly-thienylene-vinylene (PTV) and substituted polyacetylene (s$(\mathrm{CH})_{x}$ ). In addition, PA of a thiophene oligomer, $\alpha-6 \mathrm{~T}$, showing only weak photoluminescence (PL), has been measured. We demonstrate in this report that CCL can be easily used in combination with synchronously pumped dye lasers for pump-andprobe measurements and extend the probe spectral range to the $I R$ from $1.8 \mu \mathrm{m}$ to $1.4 \mu \mathrm{m}$, while preserving the temporal resolution of order $10 \mathrm{ps}$. 


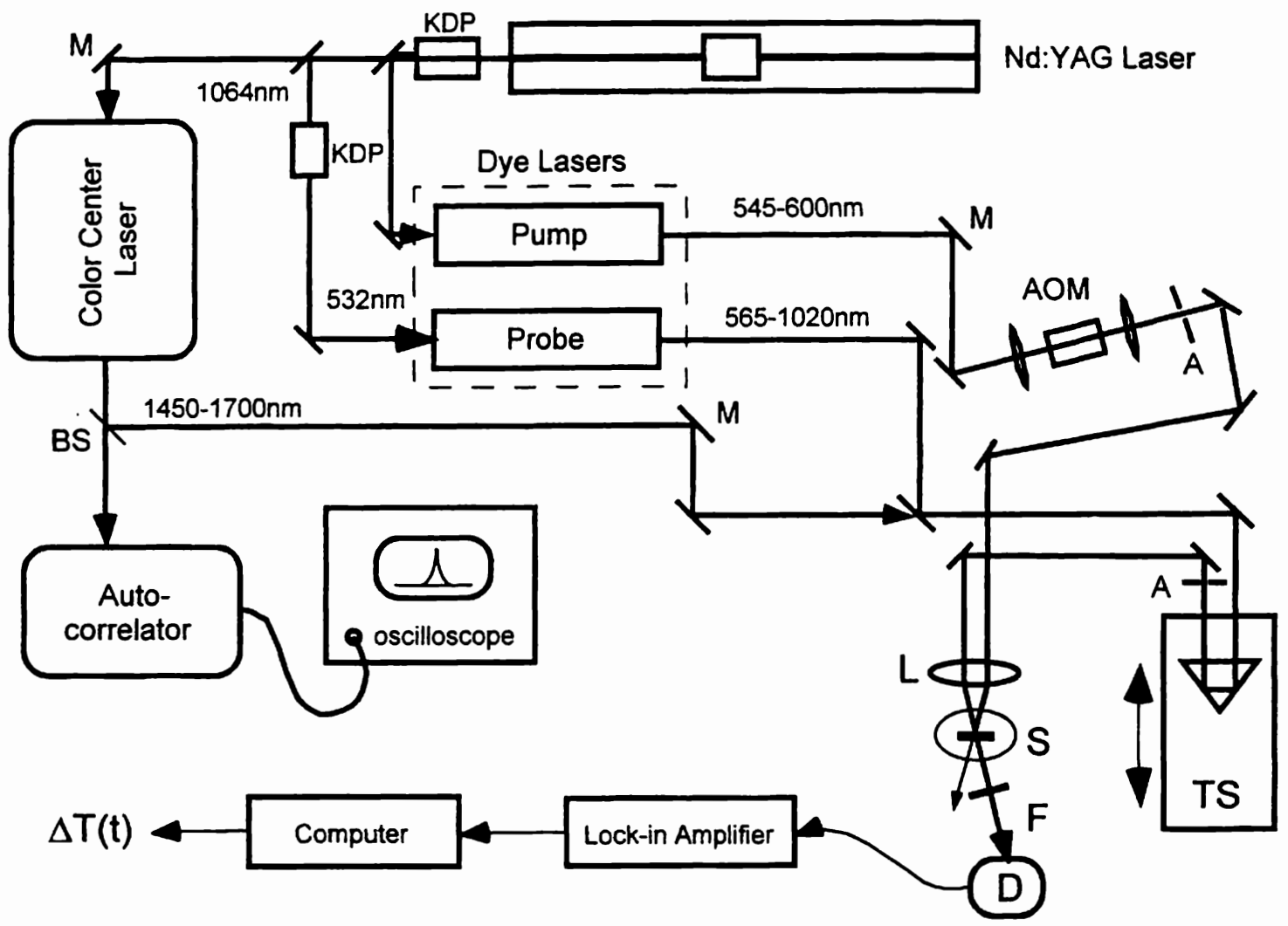

Fig.1. Time-resolved picosecond pump-and-probe experimental setup.

\section{EXPERIMENTAL APPARATUS}

In the transient pump-and-probe photomodulation (PM) technique the change $\Delta \mathrm{T}$ in the probe beam transmission, $\mathrm{T}$, is measured at a delay time $t$ following the initial photoexcitation with a pump beam. Transient PM spectra have been obtained using ps pulsed, synchronously pumped by a Nd:YAG laser, dye lasers and a CCL (Fig.1). The pump beam has been produced by one of the dye lasers with fixed wavelength at $570 \mathrm{~nm}$. The probe beam wavelength could be varied between $570 \mathrm{~nm}$ and $1020 \mathrm{~nm}$ using the second dye laser, or between $1450 \mathrm{~nm}$ and $1700 \mathrm{~nm}$ using the CCL. ${ }^{3,4}$ The resulting time resolution has been determined by the pump-probe cross-correlation to be about $10 \mathrm{ps,} \mathrm{varying} \mathrm{with} \mathrm{the} \mathrm{probe} \mathrm{wavelength.}$ Using fast modulation $\left(4 \mathrm{MHz}\right.$ ) and phase sensitive lock-in detection, ${ }^{4} \Delta \mathrm{T} / \mathrm{T}$ as small as $10^{-7}$ could be measured at photoexcitation densities of about $10^{16} \mathrm{~cm}^{-3}$ or less.

Thin polymer films of different thickness have been obtained by evaporation on quartz and sapphire substrates from polymer solutions in an appropriate solvent (toluene, chloroform, etc.). All measurements have been conducted on samples kept at $300 \mathrm{~K}$ in a dynamic vacuum in order to avoid strong photooxidation.

\section{RESULTS}

Time-resolved photomodulation (PM) spectroscopy of LCP using CCL revealed a strong PA band $\left(\mathrm{PA}_{1}\right)$ in the IR range of $0.8-1.0 \mathrm{eV}$ as shown in Fig.2(a,b,c) for three different LCP films. ${ }^{5}$ Previously measured stimulated emission (SE) band above $1.7 \mathrm{eV}$ and $\mathrm{PA}$ band at $1.5 \mathrm{eV}\left(\mathrm{PA}_{2}\right)$ have been attributed to emissive excitons, which are considered to be the primary photoexcitations in conducting polymers. ${ }^{6} \mathrm{~A}$ similar assignment can be made for $\mathrm{PA}_{1}$, comparing the decay dynamics of $\mathrm{PA}_{1}$ 

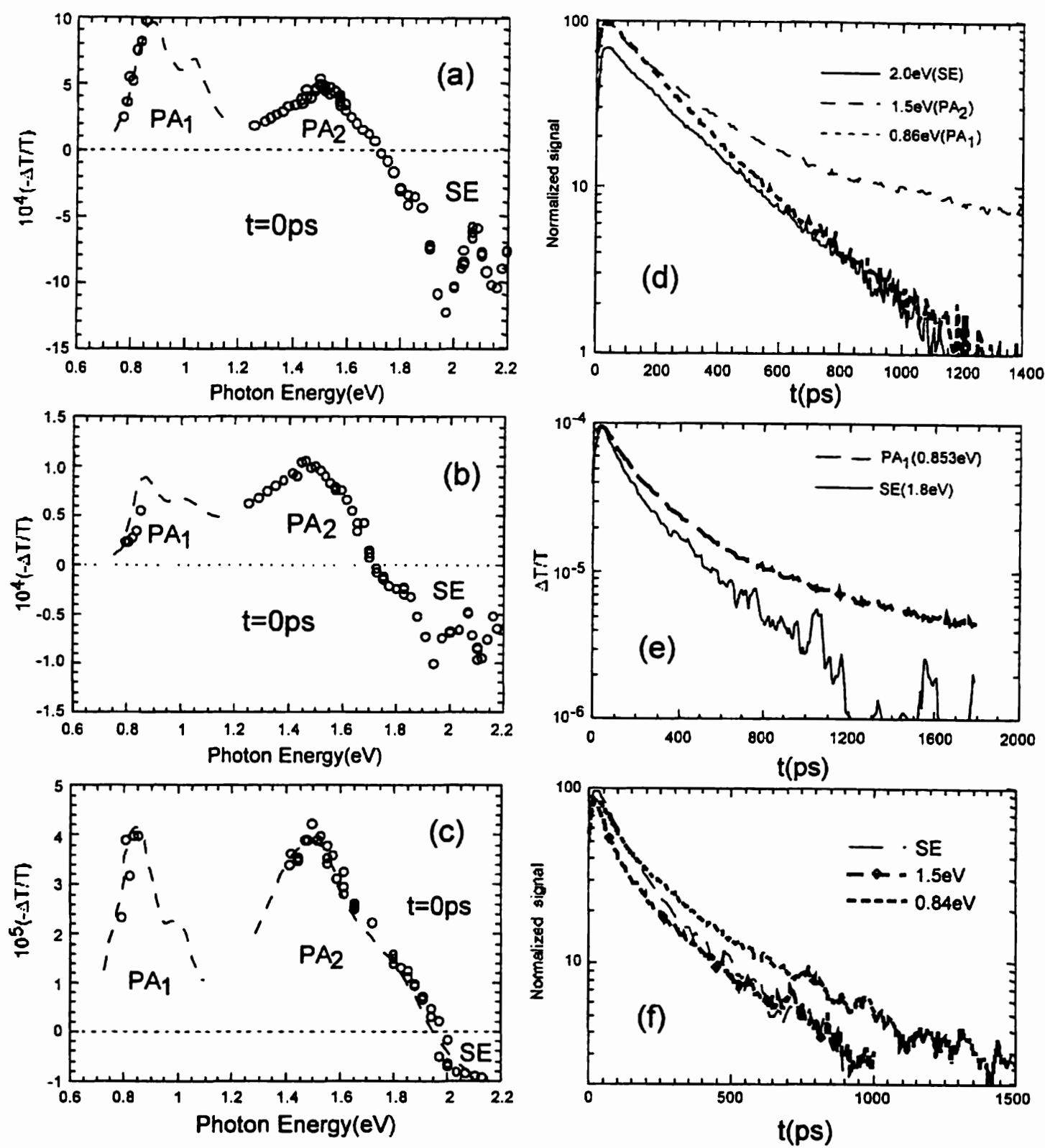

Fig.2. Transient PM spectra at t=0ps in DOO-PPV (a), P3BT (b), PPE (c), and PM decay dynamics in DOO-PPV (d), P3BT (e) and PPE (f).

and SE. Fig.2(d,e,f) show that $\mathrm{PA}_{1}$ and SE decays are very well correlated, and since SE is due to single excitons, we may attribute $\mathrm{PA}_{1}$ to singlet excitons as well. We note that this is a common feature in the three different LCP studied here, where the transient PM spectrum consists of two strong excitonic PA bands and a SE band.

Results of PM measurements in $\alpha-6 \mathrm{~T}$ films are given in Fig.3. The PL yield of $\alpha 6 \mathrm{~T}$ is very small, indicating that the primary excitations in this material are not emissive. However, as in LCP films, the PM spectrum of $\alpha-6 \mathrm{~T}$ still contains a characteristic feature of singlet excitons - two $\mathrm{PA}$ bands $\left(\mathrm{PA}_{1}\right.$ and $\left.\mathrm{PA}_{2}\right)$ as shown in Fig.3(a). In addition, there is a strong long-lived $\mathrm{PA}$ band at $1.6 \mathrm{eV}$, which has been assigned to polaron pairs. ${ }^{7} \mathrm{PA}_{1}$ and $\mathrm{PA}_{2}$ decay dynamics are compared in Fig.3(b), which shows that they are due to the same species. In addition, the PL decay is also shown, which does not follow 

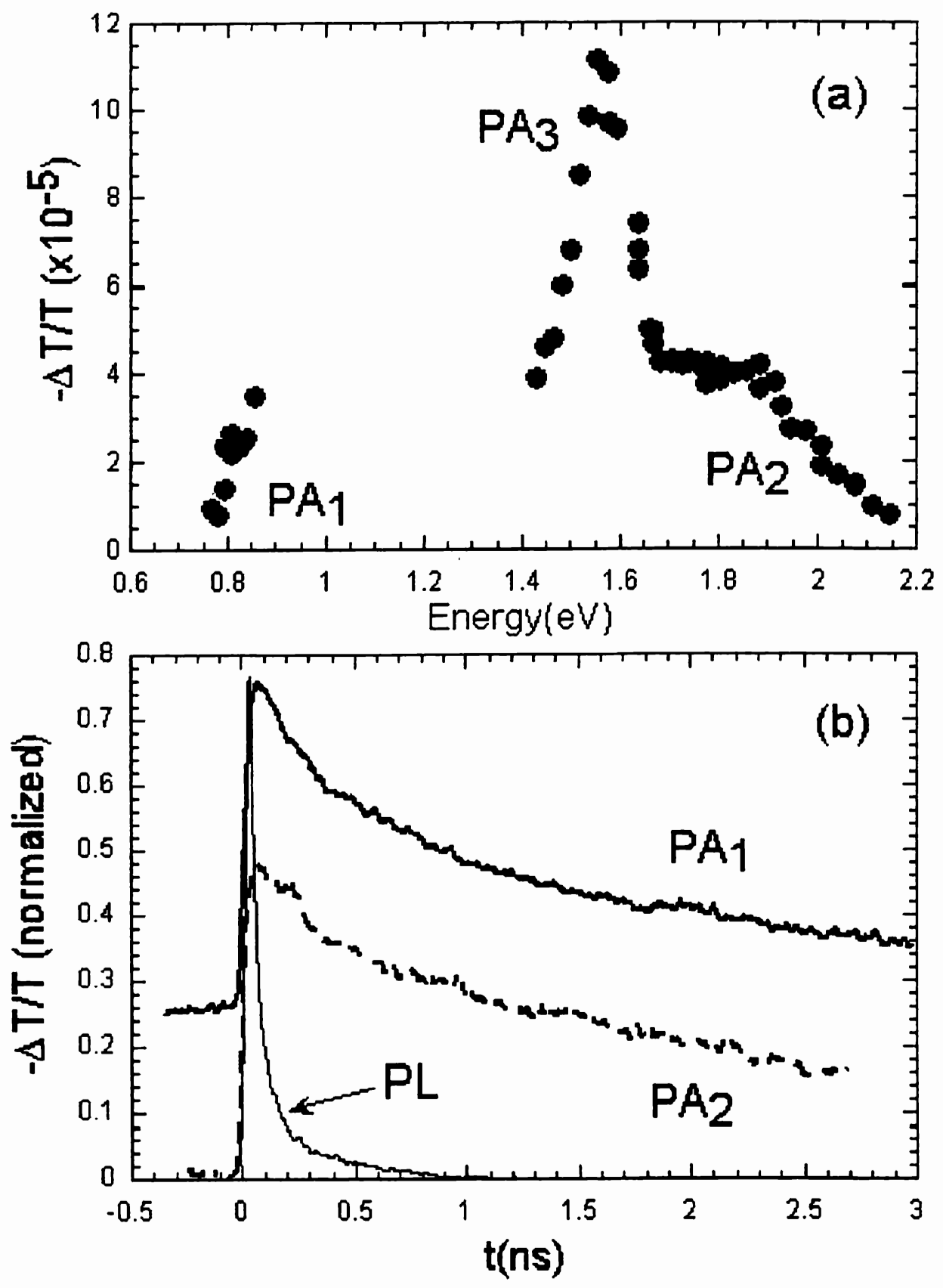

Fig.3. Transient PM spectrum at $t=0 p s$ (a) and its decay (b) in $\alpha-6 \mathrm{~T}$ films.

the PA decays. We thus attribute these PA bands to nonemissive singlet excitons, the lowest excited state of which is of even parity and therefore optically forbidden.?

The transient PM spectra of our NCP films are shown in Fig.4(a,b). They are substantially broader than those of LCP films. However, we might be able to separate several characteristic $P A$ bands, such as $P_{1}, P A_{2}$, and $P_{3}$, as marked in Fig.4. Since NCP are nonemisive, there is no direct proof that their primary photoexcitations are excitons. It is thought that the lowest energy exciton in NCP is of even parity $\left(2 \mathrm{~A}_{\mathrm{g}}\right)$ and therefore cannot decay radiatively. Thus excitonic PA might be the only way to monitor the exciton decay dynamics in NCPs. We consider $P A_{1}$ in the IR range to be a good candidate for probing nonemissive excitons. Fig.4(c,d) show the corresponding $\mathrm{PA}_{1}$ decays for two different NCP films. $\mathrm{PA}_{1}$ decay in 
$s-(C H)_{x}$ is much faster than that in PTV. In fact, the excitation lifetime in $s-(C H)_{x}$ is shorter $(\sim 1 \mathrm{ps})$ than the pulsewidths of the pump and probe beams, ${ }^{4}$ so that the photoresponse given in Fig.4(c) can be used as a cross-correlation function to determine the time resolution of the picosecond pump-and-probe measurements.
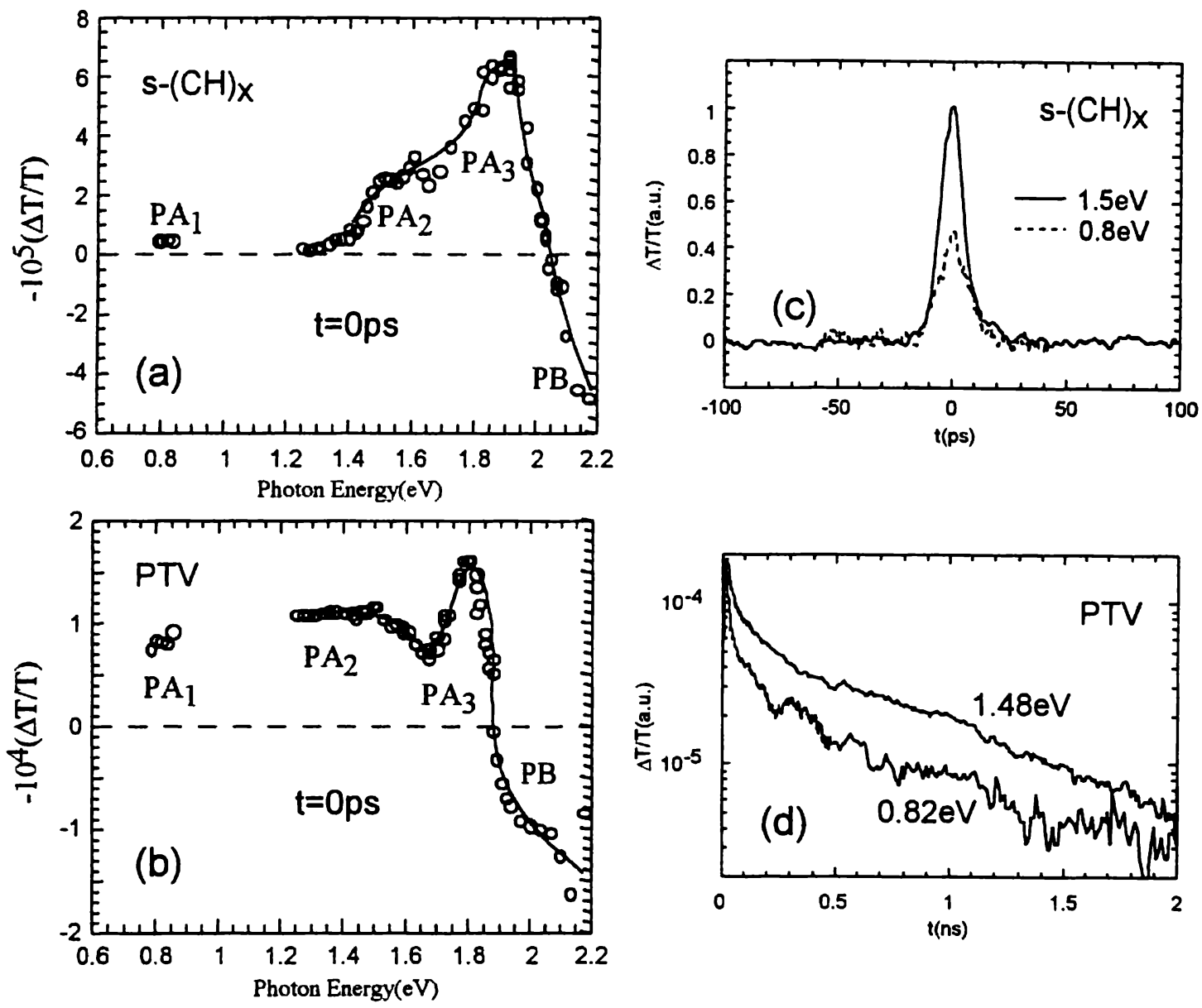

Fig.4. Transient PM spectra of s-(CH) $)_{x}(a)$ and PTV(b) and their respective PM dynamics (c,d).

\section{DISCUSSION}

The IR PA 1 band in LCP films can be uniquely associated with emissive singlet excitons by comparing its dynamics with the decay dynamics of either PL or SE. A similar comparison, however, is not possible in $\alpha 6 \mathrm{~T}$ and NCP films. On the other hand, it is very tempting to consider $\mathrm{PA}_{1}$ as a general signature of singlet excitons, which is independent of their particular parity (odd for emissive excitons, even for nonemissive excitons) ${ }^{8}$. Nevertheless, proving excitonic nature of $\mathrm{PA}_{1}$ in NCP requires additional experimental evidence. Fig. 5 schematically shows the proposed assignment of $\mathrm{PA}_{1}$ and $\mathrm{PA}_{2}$ bands to singlet exciton transitions for both LCP and NCP.

In summary, we have shown that a color center laser can be successfully used in the pump-and-probe measurements together with regular, synchronously pumped dye lasers. CCL can cover an $\mathbb{R}$ spectral range around $0.8 \mathrm{eV}$, which turns out to be an important range for spectroscopic PM studies. We have demonstrated that $\pi$-conjugated systems, including 
luminescent and nonluminescent polymers and an important oligomer ( $\alpha-6 \mathrm{~T})$, exhibit a strong PA band in this IR range. This band in LCP can be shown to be due to singlet excitons, and by analogy, it may be attributed to similar species in NCP.

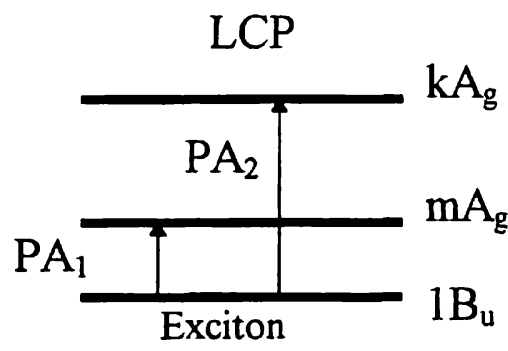

Ground state $1 \mathrm{~A}_{\mathrm{g}}$

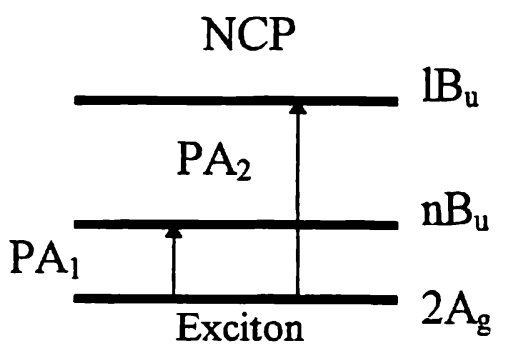

Ground state $1 \mathrm{~A}_{\mathrm{g}}$

Fig.5. Exciton transitions in the singlet manifold for both LCP (left) and NCP (right) and their assignment to PA bands in the transient PM spectra.

\section{ACKNOWLEDGEMENTS}

This work was supported in part by the DOE, FG-03-96-ER45490.

\section{REFERENCES}

1. "Physical Properties of Polymers Handbook", edited by J.E.Mark (AIP Press, Woodbury, NY, 1996); Proc. Int. Conf. on Science and Technology of Synth. Metals, edited by Z.V.Vardeny and A.J.Epstein (Snowbird, Utah, 1996) (Synth. Met. 84-86, 1997).

2. P.Lane, X.Wei and Z.V.Vardeny, Phys. Rev. B (in press).

3. W. Gellermann, J. Phys. Chem. Solids 52, 249 (1991).

4. S.V.Frolov, PhD dissertation, University of Utah, 1996 (unpublished).

5. We note that in this study the probe spectral range was extended to cover only the low energy side of $P A_{1}$.

6. S.V. Frolov et al., Phys. Rev. Lett. 78, 4285 (1997).

7. G.Lanzani et al., Phys. Rev. Lett. (to be published).

8. Z.G.Soos et al., Phys. Rev. Lett. 71, 1609 (1993); M.Chandross et al., Phys. Rev. B 50, 14702 (1994). 